\title{
Efecto de sustratos con compost y fertilización nitrogenada sobre la fotosíntesis, precocidad y rendimiento de pimiento (Capsicum annuum)
}

\author{
Javier De Grazia ${ }^{1}$, Pablo A. Tittonell ${ }^{2}$ y Angel Chiesa ${ }^{3}$ \\ ${ }^{1,3}$ Catedras de Edafología y Horticultura, respectivamente. Facultad de Ciencias Agrarias, \\ Universidad Nacional de Lomas de Zamora, Ruta Provincial n 4 km 2, Llavallol (1836), Buenos Aires, Argentina. \\ ${ }^{2}$ Plant Production Systems, Department of Plant Science, Wageningen University, \\ P.O. Box 430, 6700 AK Wageningen, The Netherlands.
}

\begin{abstract}
J. De Grazia, P.A. Tittonell, and A. Chiesa. 2007. The effect of substrates with compost and nitrogenous fertilization on photosynthesis, precocity and pepper (Capsicum annuum) yield. Cien. Inv. Agr. 34(3): 195-204. Fast-growing seedlings have larger immediate nutrient demands as compared with adult plants. A tray experiment was conducted to evaluate the fertilization of sweet pepper transplants growing on different substrates [Control (60\% Sphagnum peat $+40 \%$ perlite), Mix I ( $45 \%$ Sphagnum peat $+30 \%$ perlite $+25 \%$ farmyard compost $)$, Mix II ( $30 \%$ Sphagnum peat $+20 \%$ perlite $+50 \%$ farmyard compost), and Commercial ( $40 \%$ compost + Sphagnum peat + perlite + vermiculite)] with weekly applications of nitrogen $\mathrm{N}$ at rates of 150 and $300 \mathrm{mg} \cdot \mathrm{L}^{-1}$, compared to a control without fertilization. Seedlings were transplanted in a randomized, complete block design with four replications. Leaf area and fresh and dry weights of leaves, stems and roots were measured for the transplants; the leaf weight ratio, specific leaf area, absolute and relative growth rates, leaf expansion rates, leaf area duration, and net assimilation rate were calculated. Precocity, early yield, and total yield were measured for the field crop. The application of nitrogen had positive effects on most growth parameters of seedlings growing on substrates with compost, promoting increased precocity and yield in the transplanted crop. Few benefits from nitrogen fertilization were observed for seedlings growing on substrates without compost. The main effect of the latter is to improve the efficiency of capture of the applied nitrogen, due to better water retention and ion-exchange capacity.
\end{abstract}

Key words: Capsicum annuum, compost, early yield, growing medium, leaf area, nitrogen, seedling.

\section{Introducción}

La etapa inicial en el crecimiento de los plantines de pimiento (Capsicum annum L.), y otras especies hortícolas, constituye el momento más crítico para obtener una buena producción. La velocidad de emergencia, uniformidad y la tasa de crecimiento inicial son determinantes para la obtención de plantines de calidad y en períodos de tiempo razonables (De Grazia et $a l ., 2004 a)$.

Recibido 06 marzo 2007. Aceptado 03 septiembre 2007.

${ }^{1}$ Dirigir correspondencia a J. De Grazia: jadegrazia@yahoo.com
La finalidad de cualquier mezcla de sustratos utilizada en la producción de plantines florihortícolas es obtener una planta de calidad, en el período más corto y con los costos de producción más bajos que sea posible (Buyatti, 2000). Con estos propósitos, la turba de Sphagnum ha sido el material orgánico mas frecuentemente empleado como componente de los sustratos para la producción de plantines comerciales. Sin embargo, se trata de un recurso no renovable y se espera que su precio inevitablemente suba. Por consiguiente, parece indispensable obtener sustitutos para la turba Sphagnum (Subler et al., 1998).

Los plantines presentan una elevada demanda de nutrientes minerales, en parte como 
resultado de la alta tasa de crecimiento en relación con las plantas adultas (Wien, 1997). Además de representar una fuente de nutrientes, los sustratos a utilizar en la producción de plantines deben permitir una buena retención y disponibilidad de agua, promover un eficiente intercambio de gases y servir de soporte físico a la planta (Leskovar y Stoffella, 1995).

Los plantines producidos en contenedores pueden estar expuestos a distintos tipos de estrés (agua, nutrición, luz, temperatura y resistencias mecánicas), pudiendo causar daños morfológicos y/o fisiológicos durante el desarrollo inicial de los tejidos radicales y aéreos. Las propiedades físicas y químicas del suelo, o del medio de crecimiento en los sistemas de cultivo sin suelo, afectan la elongación, orientación y el patrón de ramificación de la raíz (Feldman, 1984). Cualquier estrés, en la zona radical se expresa en la parte aérea, afecta la partición de materia seca entre raíces y vástagos. Por lo tanto, afecta la productividad de la planta (Brouwer y De Wit, 1969). El equilibrio funcional entre raíces y vástagos corresponde a un crecimiento interrelacionado, en el cual los cambios en la tasa del crecimiento aéreo se expresan en la raíz y viceversa (Brouwer, 1963).

Por otra parte, un sistema radical corto y poco proliferado explora un menor volumen de suelo para la obtención de agua y nutrientes. Una menor longitud de raíces por unidad de volumen de suelo y/o una menor densidad radical requiere que las tasas de absorción de agua y nutrientes se mantengan más elevadas de lo normal para poder satisfacer las demandas de los plantines en crecimiento (Bennie, 1991).

Estudios realizados con materiales de origen orgánico, por ejemplo resaca de monte (restos de vegetación herbácea, arbustiva y arbórea), cama de pollo y/o gallina (mezcla de estiércol de pollo y/o gallina con los materiales que se usan como cama en los gallineros) y humus de lombriz, en mezcla con y sin suelo, mostraron resultados satisfactorios como sustratos de crecimiento para hortalizas (Princich et al., 1997; Valenzuela y Gallardo, 1997; Subler et al., 1998; Atiyeh et al., 2000; Rothman et al., 2000). Sin embargo, sustratos nutricionalmente muy enriquecidos pueden ser perjudiciales debido a su alto contenido de sales solubles (O’Hallorans et al., 1993; Chang et al., 1994; Chang y Rinker, 1994; Gómez et al., 1996).

En gran medida, el uso de materiales compostados permite reemplazar la utilización de recursos no renovables (ej. turba), y transformar en sustrato aprovechable desechos orgánicos que eventualmente contaminan el medio ambiente. De este modo se favorece el crecimiento de los plantines a través de un aporte de micro y macronutrientes que de otra forma deberían ser incorporados mediante fertilización (Prieto, 2005).

La incorporación de nutrientes en forma mineral en las mezclas de sustratos puede mejorar su disponibilidad inmediata, contrarrestando el efecto de inmovilización transitoria ocasionada por los componentes orgánicos del sustrato (C: $\mathrm{N}, \mathrm{C}: \mathrm{P})$. Más aun, puede disminuir o evitar la necesidad de agregar compost o suelo. Este trabajo tuvo por objetivo evaluar el efecto de la adición de nutrientes al sustrato empleado en la producción de plantines de pimiento, a través de la caracterización del aparato fotosintético, precocidad, rendimiento total y precoz.

\section{Materiales y métodos}

Este trabajo se realizó en invernaderos destinados a producción de plantines comerciales de pimiento (C. annuum) ubicados en la zona de Las Colonias, partido de Florencio Varela, en el cinturón hortícola de Buenos Aires, entre agosto de 2004 y marzo de 2005. Se utilizó un invernadero tipo capilla, con estructura de madera y cobertura de polietileno LDT (150 $\mu$ de espesor) en techo y laterales, de $30 \mathrm{~m}^{2}$ (plantinera), con 2,9 $\mathrm{m}$ de altura de cumbrera y provisto de ventilación lateral. La calefacción se realizó mediante radiadores de resistencia eléctrica.

Se utilizó pimiento híbrido Saxo F1 (Clause Semences International Inc.). Las semillas se pre-germinaron en cámara a $25^{\circ} \mathrm{C}$, y se sembraron el 13 de agosto de 2004 con 1,5 mm de radícula emergida. Las bandejas sembradas se ubicaron en mesas a 1,10 m de altura. Se regó por microaspersión. Las bandejas se desinfectaron con hipoclorito de sodio $\left(2 \mathrm{~mL} \cdot \mathrm{L}^{-1}\right)$ previo a su llenado con sustrato estéril. Posteriormente se aplicó 
semanalmente sulfato neutro de oxiquinoleína $\left(0,3 \mathrm{~g} \cdot \mathrm{L}^{-1}\right)$ y quincenales de captan $\left(2,5 \mathrm{~g} \cdot \mathrm{L}^{-1}\right)$.

\section{Tratamientos}

Los sustratos utilizados fueron: 1. Testigo (To) consistente en $60 \%$ (v/v) de turba de Sphagnum y $40 \%$ de perlita, 2. Mezcla I (MI) compuesta por un 45\% (v/v) de turba de Sphagnum, 30\% de perlita y $25 \%$ de material vegetal compostado, 3. Mezcla II (MII) constituida por $30 \%(\mathrm{v} / \mathrm{v})$ de turba de Sphagnum, $20 \%$ de perlita y $50 \%$ de material vegetal compostado y 4. Sustrato comercial (Co) (Growmix S1, Terrafertil S.A.) compuesto por turba de Sphagnum, 40\% de compost orgánico (v/v), perlita y vermiculita. Cada sustrato fue semanalmente fertilizado con urea a razón de $150 \mathrm{mg} \cdot \mathrm{L}^{-1}$ de nitrógeno (F1) y $300 \mathrm{mg} \cdot \mathrm{L}^{-1}$ de nitrógeno (F2), más un testigo sin fertilizar (F0). Estos tratamientos se realizaron junto al agua de riego durante cuatro semanas consecutivas. Se determinó la conductividad eléctrica de cada sustrato en extracto de saturación $(1: 2,5 \mathrm{v} / \mathrm{v})$ a $25^{\circ} \mathrm{C}$.

\section{Mediciones y determinaciones}

Las muestras para la caracterización del aparato fotosintético de los plantines se obtuvieron al inicio de los tratamientos de fertilización, 27 días después de la siembra (dds) y cuando en la mayoría de los tratamientos se alcanzó el tamaño de transplante (7-8 hojas verdaderas), a los 57 dds. Cada muestra consistió en cinco plantas completas por repetición. Se determinó el área foliar (AF) y el peso fresco de hojas (PFH), tallos (PFTal) y raíces (PFR). Además, se determinó el peso seco $\left(65^{\circ} \mathrm{C}\right.$ por $\left.72 \mathrm{~h}\right) \mathrm{de}$ hojas (PSH), tallos (PSTal) y raíces (PSR).

\section{Análisis del crecimiento}

Los resultados obtenidos se procesaron de acuerdo con Hunt (1978, 1982). Se calculó la proporción areal de hojas $[\mathrm{PAH}=\mathrm{AF} \cdot(\mathrm{PSH}$ $\left.+\mathrm{PSTal}^{-1}\right]$, área foliar específica (AFE = AF·PSH $)^{-1}$, tasa de crecimiento absoluta (TCA) y la tasa de expansión foliar en términos absolutos (TEFA).

Se integró la superficie fotosintéticamente activa presente en la planta en los diferentes muestreos para estimar la duración del área foliar (DAF). La tasa relativa de crecimiento (TCR) se calculó en relación con la materia fresca y seca,

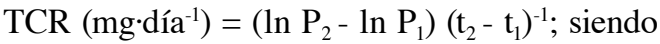
$\mathrm{P}_{2}$ y $\mathrm{P}_{1}$ los valores de los pesos respectivos a los tiempos $\mathrm{t}_{2} \mathrm{y} \mathrm{t}_{1}$ (Venus y Causton, 1979).

Mediante un procedimiento análogo se calculó la tasa de expansión foliar en términos relativos (TEFR). La eficiencia del aparato fotosintético se determinó indirectamente a través de la tasa de asimilación neta (TAN), calculada sobre la base de la relación

$\begin{aligned} \operatorname{TAN}\left(\mathrm{g}^{\circ} \mathrm{cm}^{-2} \cdot \mathrm{dia}^{-1}\right)= & \left(\mathrm{PST}_{\text {final }} / \mathrm{AF}_{\text {final }}-\mathrm{PST}_{\text {inicial }} / \mathrm{AF}_{\text {inicial }}\right) \mathrm{x} \\ & (\alpha / \alpha-1) / \mathrm{dds}_{1.2}\end{aligned}$

donde

$$
\alpha=\left(\ln \mathrm{PST}_{\text {final }}-\ln \mathrm{PST}_{\text {inicial }}\right) \cdot\left(\ln \mathrm{AF}_{\text {final }}-\ln \mathrm{AF}_{\text {inicial }}\right)^{-1}
$$

y $d d s_{1.2}$ son los días entre el primer y el segundo muestreo indicados como inicial y final en la ecuación (Evans, 1972).

En el cultivo transplantado, se determinó la precocidad en días desde el transplante (ddt) y se registró el peso de los frutos cosechados del primer racimo (el fruto de la primera ramificación dicotómica fue extraído previo a su desarrollo), considerando el momento de inicio de cosecha cuando el 50\% de las plantas de cada parcela alcanzó ese estadío. Se determinó el rendimiento a lo largo de todo el ciclo de producción.

\section{Transplante y cultivo}

El cultivo se estableció por transplante y se condujo en un invernadero de $1200 \mathrm{~m}^{2}$ con techo parabólico de 3,50 m de altura máxima y paredes a 2,10 $\mathrm{m}$. El invernadero tuvo estructura metálica con techo y cortinas de polietilenoLDT de 150 y $100 \mu$ de espesor, respectivamente, lo que permitió ventilar frontal y lateralmente. Los zócalos fueron de $60 \mathrm{~cm}$ de polietileno de $100 \mu$ de espesor.

El suelo del invernadero se trató con $10 \mathrm{~g} \cdot \mathrm{m}^{-2}$ de dazomet 20 días antes del transplante. Se practicó una fertilización base con fosfato diamónico (18:46:0) a razón de $150 \mathrm{~kg} \cdot \mathrm{ha}^{-1}$, una semana antes del transplante. El mismo 
se realizó el 23 de octubre de 2004 para todos los tratamientos, empleando un marco de plantación de 1,0 m entre líneas por 0,3 m entre plantas dentro de la línea. El invernadero no se calefaccionó y el riego se realizó por goteo.

A partir de la aparición de las primeras flores se aplicó $100 \mathrm{~kg} \cdot \mathrm{ha}^{-1}$ de urea (46:0:0) junto con el riego en cuatro aplicaciones bisemanales. El programa fitosanitario consistió en aplicaciones preventivas de los fungicidas sistémicos, benomil $\left(0,9 \mathrm{~g} \cdot \mathrm{L}^{-1}\right)$ y carbendazim $\left(0,3 \mathrm{~g} \cdot \mathrm{L}^{-1}\right)$, de contacto, captan $\left(1,2 \mathrm{~g} \cdot \mathrm{L}^{-1}\right)$ y mancozeb $\left(2 \mathrm{~g} \cdot \mathrm{L}^{-1}\right)$, y los insecticidas endosulfán $\left(1,5 \mathrm{~L} \mathrm{ha}^{-1}\right)$ y deltametrina $\left(0,2 \mathrm{~cm}^{3} \cdot \mathrm{L}^{-1}\right)$. Las malezas se controlaron manualmente.

\section{Diseño y análisis estadístico}

Durante la etapa de producción de plantines el diseño experimental empleado consistió en un arreglo completamente aleatorizado con cuatro repeticiones en combinación factorial de $3 \times 4$ factores (tres niveles de fertilización nitrogenada $x$ cuatro mezclas de sustratos), considerando a cada bandeja de 91 celdas como una unidad experimental. Los tratamientos para el cultivo trasplantado se distribuyeron conforme con un diseño de bloques completos aleatorizados con cuatro repeticiones, la unidad experimental estuvo representada por parcelas de $4,2 \mathrm{~m}^{2}$. Los resultados obtenidos fueron sometidos a análisis de varianza y los promedios se separaron de acuerdo con Tukey $(\mathrm{p}<0,05)$. La relación entre las variables de experimentación y la respuesta obtenida se estudió por análisis de regresión lineal.

\section{Resultados y discusión}

A los 27 días post siembra se obtuvieron, para los diferentes tipos de sustratos, diferencias estadísticamente significativas $(p<0,05)$ en todos los parámetros relacionados con las características del aparato fotosintético (Cuadro 1). Los plantines cultivados en el sustrato testigo mostraron los menores valores de AF, AFE, PAH y TEFA, presentando diferencias significativas $(\mathrm{p}<0,05)$ respecto de los restantes sustratos (Cuadro 1). Estos plantines resultaron ser 55,4\% del AF, 71,9\% del AFE, $79,3 \%$ de la PAH y $55,7 \%$ de la TEFA, respecto del promedio de todos los tratamientos. Esto demostró el efecto beneficioso de la adición de materiales compostados en la mezcla de sustratos para los parámetros relacionados al aparato fotosintético. El sustrato comercial no se diferenció estadísticamente de la Mezcla I en área foliar y tasa de expansión foliar absoluta. Tampoco se diferenció de la Mezcla II en área foliar específica y proporción areal de hojas, presentando mayores valores que la mezcla testigo (Cuadro 1).

En relación con la tasa de crecimiento absoluta en fresco, el sustrato comercial no se diferenció estadísticamente de la Mezcla I, pero presentó mayor tasa de crecimiento absoluta que la Mezcla II $(\mathrm{p}<0,05)$ y mayores que el sustrato testigo $(\mathrm{p}<0,01)$. La mezcla testigo produjo plantines con menor velocidad crecimiento inicial que el resto $(\mathrm{p}<0,01)$, alcanzando sólo $56,8 \%$ del valor de la TCA en fresco promedio (Cuadro 1). Este comportamiento fue diferente de lo previamente observado por Tittonell et al. (2003) y Rouphael (2004). Según estos autores los sustratos con materiales compostados o humus de lombriz producen plantines con menor velocidad de crecimiento inicial. Esto posiblemente debido a la mayor temperatura mínima de las mezclas constituidas por turba y perlita. La tasa de crecimiento absoluta, sobre la base del peso seco, mostró un comportamiento diferente, ya que los plantines cultivados en el sustrato comercial presentaron los mayores valores $(\mathrm{p}<0,01)$ y los de la Mezcla I fueron intermedios $(\mathrm{p}<0,01)$ entre aquellos y los que crecieron en la Mezcla II y el sustrato testigo (Cuadro 1).

A los 57 días post siembra, las características del aparato fotosintético evaluadas no fueron significativamente afectados por el nivel de fertilización nitrogenada aplicada (Cuadro 1). En cuanto a las mezclas utilizadas como sustrato, a los 27 dds se observaron diferencias significativas para todos los parámetros relacionados con el aparato fotosintético. Sin embargo, al momento del transplante sólo hubo diferencias estadísticamente significativas para el área foliar $(\mathrm{p}<0,10)$ entre el testigo y la Mezcla I, para la DAF entre el sustrato testigo y el resto de los sustratos $(p<0,01)$ y para la TEFR donde el sustrato testigo produjo 
Cuadro 1. Características del aparato fotosintético y tasa de crecimiento absoluta en fresco y seco de los plantines de pimiento (Capsicum annuum) al momento del inicio de los tratamientos de fertilización y características del aparato fotosintético al momento del transplante.

Table 1. Characteristics of the parameters related to the leafiness and fresh and dry absolute growth rates of pepper seedlings (Capsicum annum) at the beginning of the fertilization treatments, and characteristics of the parameters related to the "leafness" of pepper seedlings at the time of transplanting.

\begin{tabular}{|c|c|c|c|c|c|c|c|}
\hline \multirow[b]{2}{*}{ Tratamientos } & \multirow{2}{*}{$\begin{array}{l}\text { Area foliar } \\
\qquad \mathrm{cm}^{2}\end{array}$} & \multirow{2}{*}{$\begin{array}{l}\text { Area foliar } \\
\text { específica } \\
\mathrm{cm}^{2} \cdot \mathrm{g}^{-1}\end{array}$} & \multirow{2}{*}{$\begin{array}{c}\text { Proporción } \\
\text { areal de la hoja } \\
\qquad \mathrm{cm}^{2} \cdot \mathrm{g}^{-1}\end{array}$} & \multirow{2}{*}{\multicolumn{2}{|c|}{$\begin{array}{c}\text { Tasa de expansión } \\
\text { foliar absoluta } \\
\mathrm{cm}^{2} \cdot \mathrm{día}^{-1}\end{array}$}} & \multicolumn{2}{|c|}{$\begin{array}{c}\text { Tasa de crecimiento } \\
\text { absoluta, } \mathrm{mg} \cdot \mathrm{dí}^{-1}\end{array}$} \\
\hline & & & & & & en fresco & en seco \\
\hline \multicolumn{8}{|c|}{$\begin{array}{l}27 \text { días después de la siembra: } \\
\text { Sustratos: }\end{array}$} \\
\hline Testigo & $1,319 \mathrm{c}^{2}$ & $489,5 c^{2}$ & $337,2 c^{2}$ & $0,049 c^{2}$ & & $1,97 c^{2}$ & $0,23 c^{2}$ \\
\hline Mezcla I & $2,795 \mathrm{ab}$ & $625,1 \mathrm{~b}$ & $415,5 \mathrm{~b}$ & $0,104 \mathrm{ab}$ & & $4,25 \mathrm{a}$ & $0,32 \mathrm{~b}$ \\
\hline Mezcla II & $2,478 \mathrm{~b}$ & 844,9 a & $481,0 \mathrm{a}$ & $0,092 \mathrm{~b}$ & & $3,49 \mathrm{~b}$ & $0,25 \mathrm{c}$ \\
\hline \multirow[t]{2}{*}{ Comercial } & $2,925 \mathrm{a}$ & $762,5 \mathrm{a}$ & 466,9 a & 0,108 a & & $4,18 \mathrm{a}$ & $0,40 \mathrm{a}$ \\
\hline & & & & & $\begin{array}{c}\text { Tasa de } \\
\text { expansión } \\
\text { foliar relativa } \\
\mathrm{cm}^{2} \cdot \mathrm{dí}^{-1}\end{array}$ & $\begin{array}{l}\text { Duración } \\
\text { del área } \\
\text { área foliar } \\
\mathrm{cm}^{2} \cdot \text { día }\end{array}$ & $\begin{array}{c}\text { Tasa de } \\
\text { asimilación } \\
\text { neta } \\
\mathrm{mg} \cdot \mathrm{cm}^{-2} \cdot \mathrm{dí}^{-1}\end{array}$ \\
\hline \multicolumn{8}{|c|}{$\begin{array}{l}57 \text { días después de la emergencia: } \\
\text { Sustratos: }\end{array}$} \\
\hline Testigo & $7,214 \mathrm{a}^{2}$ & $1105,1 \mathrm{a}^{2}$ & $907,0 \mathrm{a}^{2}$ & $0,196 \mathrm{a}^{2}$ & $0,0530 \mathrm{a}^{2}$ & $102,30 b^{2}$ & $0,028 \mathrm{a}^{2}$ \\
\hline Mezcla I & $11,126 \mathrm{a}$ & 1180,2 a & 855,1 a & 0,278 a & 0,0451 a & $178,50 \mathrm{a}$ & $0,028 \mathrm{a}$ \\
\hline Mezcla II & 9,759 a & 1048,1 a & 775,7 a & $0,243 \mathrm{a}$ & $0,0442 \mathrm{a}$ & 157,52 a & 0,036 a \\
\hline Comercial & 9,158 a & 1019,9 a & 717,4 a & 0,208 a & 0,0367 a & $162,91 \mathrm{a}$ & 0,032 a \\
\hline \multicolumn{8}{|c|}{$\begin{array}{l}\text { Fertilización: } \\
\text { Nitrogenada, } \mathrm{mg} \cdot \mathrm{L}^{-1}\end{array}$} \\
\hline 0 & $8,902 \mathrm{a}^{2}$ & $1159,6 \mathrm{a}^{2}$ & $880,4 \mathrm{a}^{2}$ & $0,217 \mathrm{a}^{2}$ & $0,0432 \mathrm{a}^{2}$ & $146,35 \mathrm{a}^{2}$ & $0,026 \mathrm{a}^{2}$ \\
\hline 150 & $10,024 \mathrm{a}$ & 1088,7 a & 794,7 a & $0,255 \mathrm{a}$ & $0,0473 \mathrm{a}$ & 156,76 a & $0,035 \mathrm{a}$ \\
\hline 300 & 9,017 a & 1016,8 a & 766,4 a & $0,221 \mathrm{a}$ & 0,0437 a & 147,82 a & $0,032 \mathrm{a}$ \\
\hline Interación $F \times S$ & $n s$ & $n s$ & $n s$ & $n s$ & $n s$ & $n s$ & $n s$ \\
\hline \multicolumn{8}{|c|}{$\begin{array}{l}{ }^{1} \text { Testigo }(\mathrm{To})=60 \%(\mathrm{v} / \mathrm{v}) \text { de turba de Sphagnum y } 40 \% \text { de perlita, Mezcla I }(\mathrm{MI})=45 \%(\mathrm{v} / \mathrm{v}) \text { de turba de Sphagnum, } 30 \% \text { de perlita y } 25 \% \\
\text { de material vegetal compostado, Mezcla II (MII) }=30 \%(\mathrm{v} / \mathrm{v}) \text { de turba de Sphagnum, } 20 \% \text { de perlita y } 50 \% \text { de material vegetal compostado y } \\
\text { Comercial }(\mathrm{Co})=\text { Growmix S1 (Terrafertil S.A.) compuesto por turba de Sphagnum, } 40 \% \text { de compost orgánico }(\mathrm{v} / \mathrm{v}) \text {, perlita y vermiculita. } \\
{ }^{2} \text { Promedios de cinco platines por repetición seguidos de letras distintas difieren significativamente entre sí según Tukey }(\mathrm{p}<0,05) \text {. } \\
{ }^{2} \text { Control }(\text { To })=60 \%(\mathrm{v} / \mathrm{v}) \text { Sphagnum peat and } 40 \% \text { pearlite. Substrate mixture I }(\mathrm{MI})=45 \%(\mathrm{v} / \mathrm{v}) \text { Sphagnum peat, } 30 \% \text { pearlite and } 25 \% \\
\text { composted plant materials. Substrate mixture II }(\mathrm{MII})=30 \%(\mathrm{v} / \mathrm{v}) \text { Sphagnum peat, } 20 \% \text { pearlite, and } 50 \% \text { composted plant materials, and } \\
\text { Commercial substrate }(\text { Co })=\text { Grow Mix SI } \text { (Terrafertil S.A.) composed of Sphagnum peat, organic compost, pearlite and vermiculite. } \\
{ }^{2} \text { Means for five seedlings per replicate followed by different letters are significantly different according to Tukey }(p<0.05) \text {. }\end{array}$} \\
\hline
\end{tabular}

mayores valores que el sustrato comercial. Las otras mezclas de sustratos presentaron un nivel intermedio sin diferenciarse entre sí (Cuadro 1). En la Figura 1 se indica la disminución de TEFA de los plantines cultivados sobre el sustrato comercial en etapas más tardías del ciclo del cultivo, condicionando al resto de las variables relacionadas.

La proporción areal de hojas (PAH) no se diferenció significativamente al aumentar la proporción de compost (Cuadro 1). Efecto que se tradujo sobre la eficiencia fotosintética, por cuanto la tasa de asimilación neta (TAN) tampoco presentó diferencias estadísticas. Este comportamiento fue diferente al descrito en trabajos previos (De Grazia et al., 2001). A diferencia de lo observado en dicho trabajo donde la DAF aumentó linealmente con el incremento de la proporción de compost presente en la mezcla de cultivo, en este trabajo al aumentar el contenido de compost, la duración del área foliar presentó una función cuadrática negativa con un valor máximo entre la mezcla I y el sustrato comercial (Figura 2). 
Cuadro 2. Tasas de crecimiento absolutas y relativas en fresco y en seco de los plantines de pimiento (Capsicum annuum) al momento del transplante, 57 días post siembra.

Table 2. Fresh and dry absolute and relative growth rates of pepper seedlings (Capsicum annuum) at the time of transplanting (57 days after the sowing date).

\begin{tabular}{|c|c|c|c|c|}
\hline \multirow[b]{2}{*}{ Tratamientos } & \multicolumn{2}{|c|}{ Tasa de crecimiento absoluta } & \multicolumn{2}{|c|}{ Tasa de crecimiento relativa } \\
\hline & $\begin{array}{l}\text { en fresco } \\
\mathrm{mg} \cdot \mathrm{día}^{-1}\end{array}$ & $\begin{array}{l}\text { en seco } \\
\mathrm{mg} \cdot \mathrm{día}^{-1}\end{array}$ & $\begin{array}{l}\text { en fresco } \\
\mathrm{mg} \cdot \mathrm{día}^{-1}\end{array}$ & $\begin{array}{l}\text { en seco } \\
\mathrm{mg} \cdot \mathrm{día}^{-1}\end{array}$ \\
\hline \multicolumn{5}{|c|}{ Fertilización nitrogenada $(N), m g \cdot L^{-1}$} \\
\hline 0 & $3,96 \mathrm{a}^{1}$ & $0,15 \mathrm{a}^{1}$ & $0,0266 \mathrm{a}^{1}$ & $0,0138 \mathrm{a}^{1}$ \\
\hline 150 & $6,05 \mathrm{a}$ & $0,23 \mathrm{a}$ & $0,0349 \mathrm{a}$ & $0,0193 \mathrm{a}$ \\
\hline 300 & 5,45 a & $0,21 \mathrm{a}$ & $0,0336 \mathrm{a}$ & 0,0182 a \\
\hline \multicolumn{5}{|l|}{ Tipo de sustrato $(S)$} \\
\hline Testigo & $3,94 \mathrm{a}^{1}$ & $0,12 \mathrm{a}^{1}$ & $0,0363 \mathrm{a}^{1}$ & $0,0132 a b^{1}$ \\
\hline Mezcla I & $5,63 \mathrm{a}$ & $0,23 \mathrm{a}$ & $0,0294 \mathrm{a}$ & $0,0182 \mathrm{ab}$ \\
\hline Mezcla II & $6,13 \mathrm{a}$ & $0,27 \mathrm{a}$ & $0,0348 \mathrm{a}$ & $0,0257 \mathrm{a}$ \\
\hline Comercial & 4,93 a & $0,16 \mathrm{a}$ & $0,0263 \mathrm{a}$ & $0,0113 \mathrm{~b}$ \\
\hline Interacción $F \times S$ & $n s$ & $n s$ & $n s$ & $n s$ \\
\hline
\end{tabular}

${ }^{1}$ Medias de cinco repeticiones de un plantín cada una seguidas de letras distintas entre niveles de fertilización o entre tipos de sustrato difieren significativamente según Tukey $(\mathrm{p}<0,05)$. Interacción fertilización x sustrato no significativa (ns).

${ }^{1}$ Means for five plants per replicate followed by different letters within the groups for nitrogen fertilization and type of substrate are significantly different according to Tukey $(p<0.05)$. A non-significant $(n)$ interaction between nitrogen fertilization and type of substrate was observed.

A diferencia de lo reportado en trabajos previos (Tittonell et al., 2002), donde la TCR en seco se explicó principalmente por el crecimiento del área foliar que por una mayor tasa de asimilación neta, en este ensayo el comportamiento observado para la TCR en seco se encontraría asociado a una mayor intensidad del proceso fotosintetizante $\left(\mathrm{y}=0,6751 \mathrm{x}-0,004 ; \mathrm{r}^{2}=0,65\right.$; $\mathrm{p}<0,05)$, caracterizado por la TAN, que con el desarrollo del área foliar $(\mathrm{y}=0,0022 \mathrm{x}-0,0033$; $\left.r^{2}=0,41 ; p<0,05\right)$. Sin embargo, esta diferencia podría estar condicionada por la baja relación existente entre el AF y la TCR en seco para los plantines que no fueron fertilizados (Figura 3).

La tasa de crecimiento absoluta, tanto en base al peso fresco como seco, no se afectó significativamente ni por el nivel de fertilización nitrogenada ni por el tipo de sustrato empleado (Cuadro 2). Esto difiere con los resultados obtenidos previamente donde la TCA en base al peso seco de los plantines aumentó con el incremento en la proporción de materiales compostados en el medio de crecimiento (De Grazia et al., 2004b). La TCR en fresco no se diferenció en forma significativa ni para los niveles de fertilización ni para las diferentes mezclas empleadas. Por el contrario, la TCR en base al peso seco fue más elevada para la mezcla con mayor participación de compost (MII), mostrando un comportamiento similar a lo observado en otros trabajos (De Grazia et al., 2001).

Independientemente de la mezcla de sustratos empleada, la adición de nitrógeno al sustrato no afectó en forma significativa la precocidad de los plantines, el rendimiento precoz ni el rendimiento total (Cuadro 3). Los plantines provenientes de la mezcla testigo demoraron 3,55 días más que el promedio de los tratamientos en desarrollar los primeros frutos hasta un tamaño comercializable. Estos se diferenciaron significativamente $(\mathrm{p}<0,05)$ de los sustratos con materiales compostados. El rendimiento precoz fue significativamente inferior para el testigo respecto de la MI y MII. La producción obtenida en la primer cosecha por los plantines cultivados en la mezcla testigo sólo alcanzó 73,6\% del promedio de los tratamientos, demostrando el efecto benéfico que tuvo la adición del material compostado sobre este parámetro. Sin embargo, este efecto no se tradujo en diferencias significativas 
Cuadro 3. Precocidad, rendimiento precoz y total de las plantas de pimiento (Capsicum annuum).

Table 3. Precocity, early yield and total yield of pepper plants (Capsicum annuum).

\begin{tabular}{|c|c|c|c|}
\hline Tratamientos & $\begin{array}{c}\text { Precocidad } \\
\text { ddt }\end{array}$ & $\begin{array}{c}\text { Rendimien } \\
\text { precoz } \\
\mathrm{t} \cdot \mathrm{ha}^{-1}\end{array}$ & $\begin{array}{c}\text { (peso fresco) } \\
\text { total } \\
\mathrm{t} \cdot \mathrm{ha}^{-1}\end{array}$ \\
\hline \multicolumn{4}{|c|}{ Fertilización nitrogenada $(N), \mathrm{mg} \cdot \mathrm{kg}^{-1}$} \\
\hline 0 & $80,92 \mathrm{a}^{1}$ & $7,27 \mathrm{a}^{1}$ & $25,45 \mathrm{a}^{1}$ \\
\hline 150 & 79,33 a & 7,19 a & 26,10 a \\
\hline 300 & $78,75 \mathrm{a}$ & $7,01 \mathrm{a}$ & $26,00 \mathrm{a}$ \\
\hline \multicolumn{4}{|l|}{ Tipo de sustrato (S) } \\
\hline Testigo & $83,22 \mathrm{a}^{1}$ & $5,26 \mathrm{~b}^{1}$ & $23,19 \mathrm{a}^{1}$ \\
\hline Mezcla I & $77,11 \mathrm{~b}$ & $8,44 \mathrm{a}$ & $28,40 \mathrm{a}$ \\
\hline Mezcla II & $78,67 \mathrm{~b}$ & $7,64 \mathrm{a}$ & 26,78 a \\
\hline Comercial & $79,67 \mathrm{~b}$ & $7,27 \mathrm{ab}$ & 25,01 a \\
\hline Interacción $F \times S$ & $n s$ & $n s$ & $n s$ \\
\hline \multicolumn{4}{|c|}{$\begin{array}{l}{ }^{1} \text { Medias de cinco plantas por repetición seguidas de letras distintas entre niveles de fertilización o entre tipos de sustrato difiere } \\
\text { significativamente según Tukey }(\mathrm{p}<0,05) \text {. Interacción fertilización } \mathrm{x} \text { sustrato no significativa }(\mathrm{ns}) \text {. ddt: días desde el transplante. } \\
\text { 'Means for five plants per replicate followed by different letters within the groups for nitrogen fertilization and type of substrate ar } \\
\text { statistically significant according to Tukey }(p<0.05) \text {. A non-significant }(n s) \text { interaction between nitrogen fertilization and type of substrat } \\
\text { was observed. dft, days from transplanting. }\end{array}$} \\
\hline
\end{tabular}

respecto el rendimiento alcanzado a lo largo de todo el ciclo de producción para ninguno de los sustratos evaluados (Cuadro 3).

La fertilización nitrogenada de la mezcla de sustrato testigo fue insuficiente para que los plantínes alcanzaran los valores obtenidos al cultivarlos en las mezclas de sustratos con compost. Por lo tanto, es posible que el principal efecto benéfico de los materiales compostados sea disminuir la lixiviación de nutrientes desde la matriz del sustrato gracias a la mayor retención hídrica y al aumento de la capacidad de intercambio de iones (De Grazia et al., 2004a).

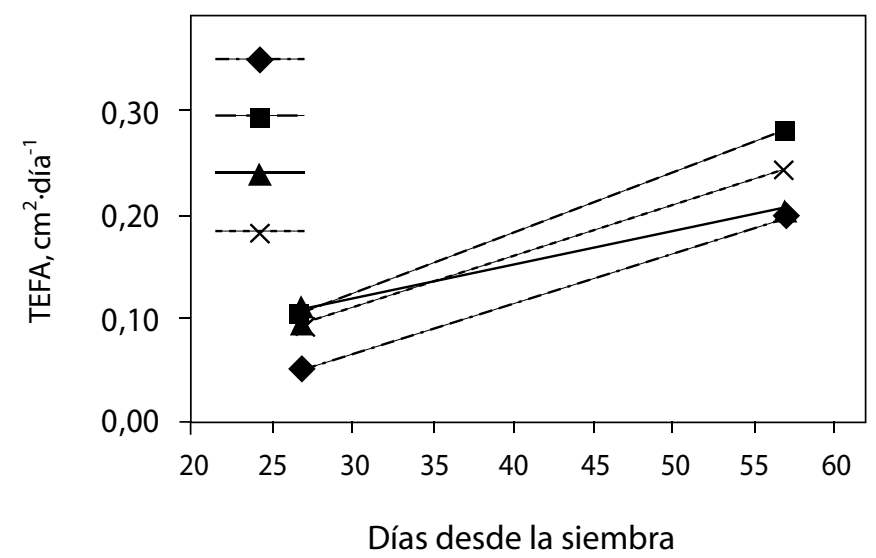

Figura 1. Evolución de la tasa de expansión foliar absoluta (TEFA) de los plantines de pimiento (Capsisum annuum) cultivados en diferentes mezclas utilizadas como sustrato. Cada punto representa el promedio de cinco plantines por repetición. To $=$ sustrato testigo, $\mathrm{MI}=$ Mezcla I, Co = sustrato comercial, MII = Mezcla II.

Figure 1. Characterization of the absolute leaf expansion rate (ALER) of pepper seedlings (Capsicum annuum) on different substrate mixtures: To = Control substrate, Co = Commercial substrate, MI = Substrate mixture I, and MII = Substrate mixture II. Each point is the mean for five seedlings per replicate. 


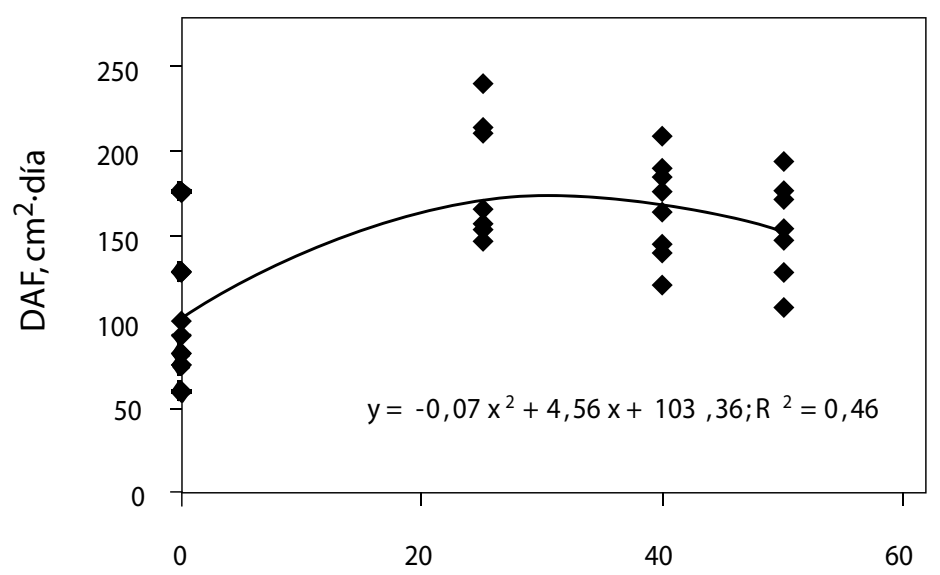

Proporción (v/v) de materiales compostados, \%

Figura 2. Relación entre la duración del área foliar (DAF) de los plantines de pimiento (Capsicum annuum) al momento del transplante y la proporción de materiales compostados presentes en el substrato de crecimiento. Cada valor representa el promedio de cinco plantines por repetición.

Figure 2. Relationship between leaf area extent (LAE) at the time of transplanting and the proportion of composted materials in the growth substrate of pepper seedlings (Capsicum annuum). Each value represents the means for five seedlings per replicate.

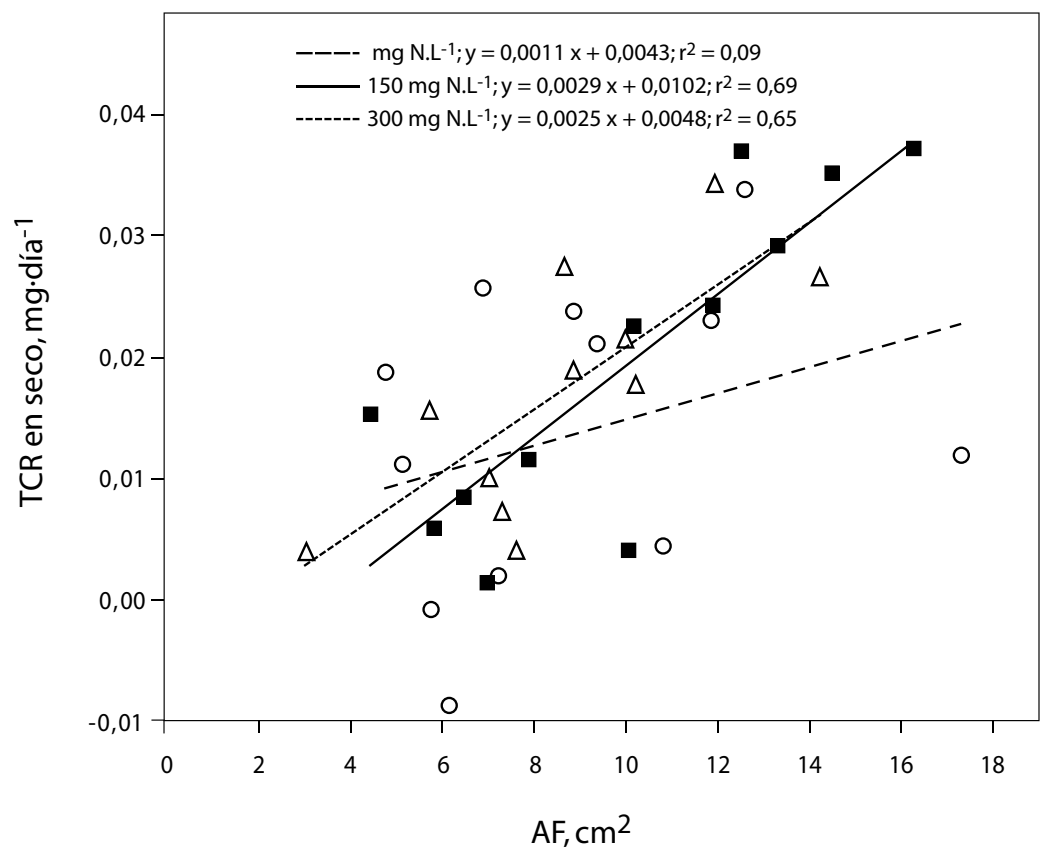

Figura 3. Relación entre la tasa de crecimiento relativa (TCR) sobre la base del peso seco y el área foliar (AF) de los plantines de pimiento (Capsicum annuиm) al momento del transplante para cada uno de los siguientes niveles de fertilización nitrogenada: $\mathrm{O}: 0 \mathrm{mg} \mathrm{N} \cdot \mathrm{L}^{-1}$; $\mathbf{D}: 150$ $\mathrm{mg} \mathrm{N} \cdot \mathrm{L}^{-1} ; \Delta: 300 \mathrm{mg} \mathrm{N} \cdot \mathrm{L}^{-1}$. Cada punto representa el promedio de cinco plantines por repetición.

Figure 3. Relationship between relative growth rate (RGR) (on a dry-weight basis) and leaf area (LA) of pepper seedlings (Capsicum annuum) at transplanting for each nitrogen fertilization level. ( $\mathrm{O}: 0 \mathrm{mg} \cdot \mathrm{L}^{-1} ; \mathbf{\square}: 150 \mathrm{mg} \cdot \mathrm{L}^{-1} ; \Delta \cdot 300 \mathrm{mg} \cdot \mathrm{L}^{-1}$ ). Each point is the mean for five seedlings per replicate. 


\section{Resumen}

Los plantines presentan elevada demanda de nutrientes como resultado de su alta tasa de crecimiento en relación a las plantas adultas. Para evaluar la adición de nitrógeno a sustratos preparados con y sin materiales compostados en la producción de pimiento, se realizó un ensayo fertilizando plantines cultivados en diferentes sustratos: Testigo (60\% turba de Sphagnum + $40 \%$ perlita), Mezcla I (45\% turba de Sphagnum + $30 \%$ perlita $+25 \%$ material vegetal compostado), Mezcla II (30\% turba de Sphagnum + 20\% perlita $+50 \%$ material vegetal compostado) y un sustrato Comercial (turba de Sphagnum $+40 \%$ compost + perlita + vermiculita) con 150 y 300 $\mathrm{mg} \cdot \mathrm{L}^{-1}$ de nitrógeno por semana, manteniendo un testigo sin fertilizar. Los plantines fueron transplantados en invernadero según un diseño de bloques completos aleatorizados con cuatro repeticiones. Previo al transplante se determinó área foliar, pesos fresco y seco de hoja, tallo y raíz, se calculó la proporción areal de hojas, área foliar específica, tasas de crecimiento absolutas y relativas en fresco y en seco, tasa de expansión foliar absoluta y relativa, duración del área foliar y tasa de asimilación neta de los plantines. En el cultivo se determinó precocidad, rendimiento precoz y total. La fertilización nitrogenada de la mezcla de sustrato testigo fue insuficiente para que los plantínes alcanzaran los valores obtenidos al cultivarlos en las mezclas de sustratos con compost. Por lo tanto, es posible que el principal efecto benéfico de los materiales compostados sea disminuir la lixiviación de nutrientes desde la matriz del sustrato gracias a la mayor retención hídrica y al aumento de la capacidad de intercambio de iones.

Palabras clave: Area foliar, Capsicum annuиm, compost, medio de crecimiento, nitrógeno, rendimiento precoz, transplante.

\section{Literatura citada}

Atiyeh, R.M., C.A. Edwards, S. Subler, and J.D. Metzger. 2000. Earthworm-processed organic wastes as components of horticultural potting media for growing marigold and vegetable seedlings. Compost Science and Utilization 8:215-223.

Bennie, A.T. 1991. Growth and mechanical impedance. Pages 393-416. In: Y. Waisel, A. Eshel, and U. Kafkafi (eds.). Plants Roots: The Hidden Half. Marcel Dekker, New York, USA.

Brouwer, R. 1963. Some aspects of the equilibrium between overground and underground plant parts. Jaarb, I.B.S. Wageningen, The Netherlands 213, 31-39.

Brouwer, R., and C.T. de Wit. 1969. A simulation model for plant growth with special attention to root growth and its consequences. Pages 224244. In: W.J. Whittington (ed.). Root Growth. Butterworths, London, UK.

Buyatti, M. 2000. Evaluación del comportamiento agronómico del aserrín de salicáceas compostado en mezcla con perlita para la producción de plantines florales. Horticultura Argentina 19:94 (Resumen 310).

Chang, C., and D.L. Rinker. 1994. Use of spent mushroom substrate for growing containerized woody ornamentals: an overview. Compost Science and Utilization 2:45-53.

Chang, C., R.A. Clinc, and D.L. Rinker. 1994. Bark and peat amended spent mushroom compost for containerized culture of shrubs. HortScience 29:781-784.

De Grazia, J., P.A. Tittonell, y A. Chiesa. 2001. Calidad y precocidad del plantín de pimiento (Capsicum annuum L.) en función de la proporción de materiales compostados presentes en el medio de cultivo. En: Actas de la II Reunión de Producción Vegetal del NOA. San Miguel de Tucumán, Tucumán, Argentina.

De Grazia, J., P.A. Tittonell, and A. Chiesa. 2004a. Growth and quality of sweet pepper (Capsicum annuum $\mathrm{L}$.) transplants as affected by substrate properties and irrigation frequency. Advances in Horticultural Sciences 18:181-187.

De Grazia, J., P.A. Tittonell, y A. Chiesa. 2004b. Eficiencia en el uso de agua en la producción de plantines de pimiento (Capsicum annuиm L.) cultivados en sustratos adicionados con polímeros superabsorbentes. Horticultura Argentina 23:22-28.

Evans, G.C. 1972. The Quantitative Analysis of Plant Growth. Blackwell Scientific Publications, Oxford, UK. 734 pp.

Feldman, L.J. 1984. Regulation of root development. Annual Review of Plant Physiology 35:223242.

Gómez, I., J. Navarro Pedreño, R. Moral, M.R. Iborra, G. Palacios, and J. Mataix. 1996. Salinity and nitrogen fertilization affecting the macronutrient content and yield of sweet pepper plants. Journal of Plant Nutrition 19:353-359.

Hunt, R. 1978. Plant Growth Analysis. Edward Arnold, London, UK. 67 pp.

Hunt, R. 1982. Plant Growth Curves. The Functional 
Approach to Plant Growth Analysis. Edward Arnold, London, UK. 248 pp.

Leskovar, D.I., and P.J. Stoffella. 1995. Vegetable seedling root systems: Morphology, development, and importance. HortScience 30:1153-1159.

O’Hallorans, J., M. Muñoz, and O. Colberg. 1993. Effect of chicken manure on chemical properties of a Mollisol and tomato production. Journal Agriculture of the University of Puerto Rico 77:181-191.

Prieto, F.S. 2005. Calidad del plantín de tomate (Lycopersicon esculentum Mill.) cultivado en sustratos adicionados con lombricompuesto. Tesis de grado. Facultad de Agronomía, Universidad de Buenos Aires, Buenos Aires, Argentina. 14 pp.

Princich, F.R., C.S. Gallardo, y O.R. Valenzuela. 1997. Empleo de lombricompuesto como sustrato de crecimiento para plantines de pimiento (Híbrido Elisa). En: Actas del XX Congreso Argentino de Horticultura. Bahía Blanca, Buenos Aires, Argentina (Resumen 23).

Rothman, S., B. Tonelli, O. Valenzuela, y M. del C. Lallana. 2000. Cultivo de plantines de tomate con sustratos basados en humus de lombriz. Horticultura Argentina 19:45 (Resumen 119).

Rouphael, Y. 2004. Yield, water requirement, nutrient uptake and fruit quality of zucchini squash grown in soil and closed soilless culture. The Journal of Horticultural Science and Biotechnology 79:423-430.

Subler, S., C. Edwards, and J. Metzger. 1998. Comparing vermicomposts and composts. BioCycle 39:63-66.

Tittonell, P.A., J. De Grazia, y A. Chiesa. 2002. Adición de polímeros superabsorbentes en el medio de crecimiento para la producción de plantines de pimiento (Capsicum annum L.). Horticultura Brasileira 20:641-645.

Tittonell, P.A., J. De Grazia, y A. Chiesa. 2003. Emergencia y tasa de crecimiento inicial en plantines de pimiento (Capsicum annuum L.) cultivados en sustratos adicionados con polímeros superabsorbentes. Revista Ceres 50:659-668.

Valenzuela, O.R., y C.S. Gallardo. 1997. Uso de lombricompuesto como medio de crecimiento para plantines de tomate (cv. Platense). Revista Científica Agropecuaria 1:15-21.

Venus, J.C., and D.R. Causton. 1979. Plant growth analysis: a re-examination of the methods of calculation of relative growth and net assimilation rates without using fitted functions. Annals of Botany 43:633-638.

Wien, H.C. 1997. Transplanting. Pages 37-67. In: The Physiology of Vegetable Crops. H.C. Wien (ed.). CAB International, Oxon, UK. 